\title{
Review on Development of Fault Diagnosis for Gyroscope
}

\author{
He SONG ${ }^{1}$, Shao-Lin HU ${ }^{1,2, a}$, and Ke-Yi ZHOU ${ }^{1}$ \\ ${ }^{1}$ School of automation and Information Engineering, Xi'an University of Technology, 710048, Xi'an, China \\ ${ }^{2}$ Foshan University, 528000, Foshan, China
}

\begin{abstract}
Gyroscope is a core part of navigation system, whose working stability and reliability directly affects the navigation performance of navigation system. Therefore, in order to ensure the precision and reliability of navigation system, it is very important to develop the techniques of fault diagnosis for gyroscope. First, the development of gyroscope's history and the background and significance of fault diagnosis for gyroscope is introduced in this paper. Then, the influence factors of abnormal faults in the running of gyroscope are analyzed in this paper, and the faults are classified into different types according to the feature of faults for gyroscope. Second, there are some main algorithms of fault diagnosis for gyroscope, such as support vector machine, wavelet analysis and neural network etc. Finally, aiming at the development status of fault diagnosis for gyroscope, the current existing problems of fault diagnosis for gyroscope are summarized, and the future development and application of fault diagnosis for gyroscope are prospected.
\end{abstract}

\section{Introduction}

Gyroscope had already experienced four stages in the development of 100 years since it was first used in ship for orientation in 1910. The first stage was the development of the ball bearing gyroscope motor and gimbaled gyroscope, which used bearing or gimbals to support high-speed rotor rotating shaft steadily measure the angular position of the carrier, and were widely applied in the aviation gyroscope instrument and flight control system, but existing outer disturbing torque made its precision decreased. The second stage was the development of the liquid floated gyroscope and gas floated gyroscope in the late 1940 s to the early 50 s, which made the framework a thin wall airtight float, using liquid or gas support high-speed rotor to decrease the friction torque and improve accuracy, but it needed the high working accuracy and cost. The third stage was the development of the rotor gyroscope with dry power flexible support in the 1960s, using the flexible junction instead of gyroscope gimbals ring and precise shaft to drive high-speed rotor without floating fluid and temperature control system, getting widely application in the aircraft and tactical missile, but it could not bear a larger impaction and vibration because the thickness of flexible support was only zero a few millimeters. The fourth stage was the rapid development of static electricity, laser, optical fiber and vibration gyroscope[1], using electrostatic or optoelectronic technology to support high-speed rotor, which had been widely used in military and civil fields such as aerospace with high precision, small volume, impact resistance and strong practicability, and would become the research and development direction of gyroscope in the future.

\footnotetext{
${ }^{a}$ Corresponding Author: Hu Shaolin, hfkth@126.com
} 
The inertia navigation system was a technology of autonomous navigation and developed in the middle of twentieth century, whose characteristics - full autonomy, all-weather, anti-disturbance, concealment and output a great number of real-time information - had not only led to provide the complete parameters(position, angular velocity and attitude angle) of navigation guidance and hence to widespread applied in the field of aerospace navigation, but had also selected as a standard and must to be installed navigation equipment. Especially, the increasing complex and intense relationship of national at present-day, the increasing dependence and requirement of inertia navigation technology changed more and more higher, and therefore it had become the key supporting technology in the competition of modern high-technology, and had the important significance to build the defense and security of national in strategic and practical.

As the core part of inertia navigation system, gyroscope can accurately determine the location of carriers and its reliability directly affects the navigation performance. According to the relevant statistics, approximately per sixty of fault is related to gyroscope in the inertia navigation system[2]. Therefore, it is great significance to ensure the safety and stability of carrier if the status of the performance of gyroscope had been judged in advance, and then timely accomplished fault diagnosis, prediction and remove for gyroscope.

\section{Fault diagnosis for gyroscope}

\subsection{Classification of fault for gyroscope}

The fault factors of gyroscope were divided into internal factors and external factors in the operational process. Internal factors mainly derived from the gyroscope itself, such as restriction of technological structure, the selection of component and the irrationality of circuit design. External factors is that the operating environment of gyroscope was relatively complex and poor, which made it easily suffer to the impaction of temperature, humidity, vibration and shock, electrical stress and other factors. For example, temperature factors can lead to gyroscope insulation aging, tensile strength aging, thermal decomposition and deformation failure. Humidity factor can cause gyroscope electrical short circuit, circuit board corrosion, surface damage and gyro motor stuck and other failures. Vibration and shock factors[3] can result in component fatigue fracture, poor contact and loose parts of the gyroscope. High-intensity and high-bandwidth radio interference can cause performance degradation and device failure and other failures.

Gyroscope will produce different patterns of abnormal failure due to the different fault inducing factors during operation. Based on the time length of failure, it may be classified into permanent failure with unrecovered, specifically as hardware damage or aging; irregular repeat intermittent failure resulting from poor contact between elements and bad environment; transient failure caused by surroundings change, such as exterior pressure change or electronic interference. Based on development degree of failure, it might be classified into unable to monitor and predict the mutability of fault in advance which could be easily checked and located, for example elements don't work suddenly; gradually failure (soft failure) varying by time and environment, such as the ones out of control leaded by decrease of accuracy and the degradation of performance.

Also, according to the common fault of gyroscope, many experts and scholars will be divided them into total failure, bias failure, drift failure and periodic disturbance failure[4], or according to the different characteristic of gyroscope output signal, the fault types can be considered as periodic failure, spike failure, pulse failure, offset failure and drift failure[5]. Despite the different types and classification of the fault for gyroscope, the occurrence of fault will lead to the reduction of accuracy and the performance degradation of gyroscope, and even affect its working safety and reliability. Therefore, it is important for ensuring the safe operation of gyroscope to investigate and seek an arithmetic which could timely, accurately and effectively deal with the detection, diagnosis and prediction of fault for gyroscope. 


\subsection{Fault diagnosis for gyroscope}

At present, although the research of fault diagnosis for gyroscope had already made great progress, the key of the research is focused on the gradual fault diagnosis caused by gyroscope drift, and thus a number of different types of algorithms of fault diagnosis for gyroscope were proposed. If summarized in accordance with the structure of algorithms, it can be divided into the following categories:

\subsubsection{Algorithms of fault diagnosis based on experience knowledge method for gyroscope}

The algorithms of fault diagnosis based on experience knowledge method for gyroscope do not need to establish the mathematical model accurately, which can make full use of expert knowledge diagnose the fault of gyroscope by introducing the information of fault for gyroscope[6]. Currently used gyroscope monitoring methods are adequate for detecting failures that can happen in the shortterm, to detect the performance degradation tendency of long-term fault, a fault detection model for gyroscope based on a fuzzy expert system solution is described and can improve the current operational processes of monitoring the gyroscope by detecting earlier on potential failures[7]; on the basis of the sequential adaptive learning algorithm, an improved evolving belief-rule-based learning algorithm based on the belief-incomplete criterion is proposed and used in the prediction of fault for gyroscope, and finally verify the validity of the algorithm and improve the precision of fault forecasting[8]; due to the existing method cannot evaluate the life of gyroscope quickly and exactly, a acceleration life evaluation method for gyroscope under temperature based on fault tree analysis is proposed and the steps of proposed method are simple and feasible[9]; in order to meet the reliability requirement of gyroscope, the problem of fault diagnosis need to be discussed and a expert system for gyroscope fault diagnosis based on Lab VIEW and Access is presented, which build the knowledge base and reasoning machine of expert system and write the corresponding software programme, the results show that the expert system can diagnose the location and reason of fault rapidly and accurately[10].

\subsubsection{Algorithm of fault diagnosis based on support vector machines for gyroscope}

Support vector machine(SVM)[11] is a new optimization algorithm which proposed by Vapnik and based on the principle of statistical learning theory and structural risk minimization, as the algorithm provided the solution of small sample, high dimension and nonlinear so that it is applied in fault diagnosis for gyroscope. A forgetting-factor least square SVM algorithm is given to build the forecasting model of drift-error-parameter of gyroscope based on the nonlinear measurement data, which used forgetting-factor to set different level of new-and-old sample and hence to avoid new information being covered by old information and enhance its self-adaptability and forecasting accuracy[12]; taking the time series of gyroscope's drift as study object, a prediction model based support vector regression is established and a pre-selecting algorithm based on the immune clustering in kernel function space is presented, and finally the method is rapid, high accuracy and practical[13], however, for improving the operational accuracy of gyroscope, the random drift error model based on wavelet analysis and least square SVM(LSSVM) is established to compensate the precision of nonlinear and weak-stability of gyroscope's random drift signal[14]; at the same time, in order to improve the learning performance and generalization ability of LSSVM, using CPSO algorithm to generate the initial chaotic particles and chaotic interrupt is added for the selection and optimization of the LSSVM, and then build the prediction model of gyroscope random drift and improve the precision of fault diagnosis[15]; for forecasting the tendency of gyroscope drift, a least square support vector regression model by introducing the wavelet kernel is presented and through genetic algorithm to optimize the model parameters, so its early forecasting ability of fault is improved and strengthened[16]; because of existing output error of gyroscope online, the online incremental LSSVM is utilized to build the dynamic model in order to obtain the real-time residual between real 
value and pre-estimating value, and then accomplish fast and accuracy online diagnosis[17]; to solve the classification accuracy reducing problem of SVM resulting from unbalanced samples and high noise of gyroscope output signals, an unbalanced FSVM fault diagnosis method of gyroscope based on DSP is presented, which can improve the diagnostic precision of fault samples and the disturbance of noise[18].

\subsubsection{Algorithms of fault diagnosis based on wavelet analysis for gyroscope}

The output signal of gyroscope is non-stationary, whose amplitude and frequency changed with the time. For these time varying signals, it is necessary to use the time-frequency analysis method which reflects its characteristics to analyze effectively and finds the fault signal. Wavelet transform can decompose the signal into multi-scale components, and selects the appropriate time domain sample step accordance with the different size of scale component to constantly focus on any small details of the processed signal[19]. For developing the model of gyroscope drift character, the multi-resolution theory of wavelet analysis is used to analyze and pretreated the signal of gyroscope drift and the result confirms its effectiveness[20]; aimed to the error fault of gyroscope drift, wavelet analysis is used to extract the error feature vector and then three-layers feedforward BP neural network is adapted to build fault model, finally fuzzy logic judgment makes diagnostic method more simple and quick[21]; Liu Luyuan extracted the tendency of the signal of gyroscope drift by the multi-resolution theory of wavelet analysis, and established the error model of gyroscope to improve the precision of model[22]; because gyroscope's drift output signal is weak and non-stationary random series, wavelet analysis is used to accomplish the hierarchical process of output data in frequency domain and use time series analysis method to build the model of gyroscope drift and finally improved the speed and precision of model[23]; according to the fault of gyroscope drift, a new prediction algorithm based on wavelet support vector machine is put forword for fault prediction and enhances the precision of fault forecasting while ensuring the safety of devices[24]; because of the mutation of the output signal of gyroscope, the signal is decomposed by three-layer wavelet package and extracts its feature to train RBF neural network, the result shows that the method can detect the fault accurately[25].

\subsubsection{Algorithms of fault diagnosis based on neural network for gyroscope}

Actually, the usage of neural network in fault diagnosis is achieve the classification of fault information without mathematical model, and adjusts the weight constantly while training to meet the requirement of precision in order to accomplish the nonlinear mapping between input and output and have the ability of self-organization and pattern recognition[26]. To solve the problem of gyroscope servo loop, the fault diagnosis using BP neural network and its software package had been simulated, the result is very satisfactory[27]; compared with BP algorithm, the LMBP algorithm which through optimizing standard BP algorithm with Levenberg-Marquardt(L-M) algorithm which had secondorder convergence effects can improve the converges of neural network learning and the ability of fault prediction for gyroscope[28]; in order to accurately and reliably detect and predict the fault of gyroscope, a wavelet based on RBF neural network fault detect algorithm is proposed, which makes the output signal decomposed into eight nodes in different frequency bands and extracts feature, then to train RBF neural network and the result shows that it can accurately diagnose the type of fault and improve the precision of fault diagnosis[29]; for discerning in real time and predicting the tendency of gyroscope drift, wavelet extraction algorithm combined with neural network nonlinear prediction algorithm which can set up the fault prediction model, and hence to reduce the impaction of environment factors and improve the precision of fault prediction[30]; because the parameter of the performance of gyroscope had degradation, a new algorithm which uses the SOFM neural network to implement the feature information fusion for charactering its degradation and uses the HMM to transform the early weak fault signals into the easily observed information, thus predicts the faults of gyroscope and the results shows that the algorithm can easily and effectively extract the feature information on gyroscope's degradation[31]; according to the fault characteristics of gyroscope, 
Hilbert-Huang Transform method is adapted to extract the feature of fault information and then uses fuzzy Kohonen neural network to build fault diagnosis model and finally improve the accuracy of fault diagnosis for gyroscope[32].

\subsubsection{Other fusion algorithms of fault diagnosis for gyroscope}

Of course, there are many other types of fusion algorithms of fault diagnosis for gyroscope. Aiming to the characteristics of gyroscope's fault, power spectra method is used to analyze the output signal eigenvalues whether fault occur or not, and then build the signal's AR model and power spectra chart so as to identify the classification of fault for gyroscope[33]; According to the tendency of gyroscope drift, a hybrid modeling method based on grey theory and stationary time series analysis is proposed and used in gyroscope drift forecasting, which has a higher forecasting precision ${ }^{[34]}$; Wang Jia has already use parameter identification method to complete performance analysis of gyroscope and the result shows that the method is very well[35]; in order to accomplish the problem of fault detection for gyroscope, an algorithm based on the sliding window least square method is designed and it can determine the fault type and fault time accurately[36]; to diagnose the fault for gyroscope when the redundancy of gyroscope cannot meet the requirement of odd-even equation, the nonlinear extended Kalman filter is adopted to estimate angular rate and can diagnose both abrupt and slow change fault of gyroscope[37].

\section{Conclusions}

In this paper, the mainstream algorithm of fault diagnosis for gyroscope is described in detail, but there are still many problems needed to be solved. First, fault diagnosis for gyroscope has a low degree of autonomy, and now most of algorithms of fault diagnosis still need researchers to identify in order to fail to achieve autonomous learning and diagnosis of fault completely. Secondly, the algorithm of fault diagnosis mainly researches the problem of single fault at present, if multiple faults occur, it is need to study how to classify and diagnose. Finally, in the big data environment, the investigation of fault diagnosis for gyroscope is just in the initial stage, and not makes great progress.

In recent years, the technology of machine learning and deep learning has developed quickly under the background of big data, which simulate the hierarchical structure of brain in order to obtain the multi-level characteristic expression from massive data, so as to improve the accuracy of classification and prediction. Therefore, the development of machine learning and deep learning will be the key technology in the research of fault diagnosis for gyroscope in the future.

\section{Acknowledgement}

This work is supported by National Natural Science Foundation of China under Grant 61473222 and Grant 91646108.

\section{References}

1. G.T. Liang, Aero. Miss. J., (4), 38-40 (2006)

2. X. Z. Guo, Theory of Gyroscope in Inertia Navigation System, (1996)

3. Chen W, Xie Q, Luo Y. Com. Inf. Sci. (ICCIS) Int. Con. IEEE, 222-225 (2010)

4. Heredia G, Ollero A. IEEE Int. Con. Rob. Auto., 2, 2229 (2005)

5. H.M. Qian, J.C. Ma, J. Elec. Meas. Ins., 21, 538-541 (2007)

6. B. Yan, Q. Wei, Y. Li, Sci. Tec. Inf., (31), 26-27 (2011)

7. A. Pereira, F. Moura-Pires, R.A. Ribeiro, ESM, 200-203 (2002)

8. X.S. Shi, C.H. Hu, Con. The. Appl., 12, 1579-1586(2012)

9. L.X. Yu, L. Qin, J. Dete. \& Con., 03, 78-81 (2015) 
10. X.D. Qin, Y.S. Li, Sci. \& Tec. Vis., 21, 110-111 (2016)

11. V. Vapnik, The nature of statistical learning theory, (Spr. Sci. \& Bus. Med., 2013)

12. W. Zhang, C.H. Hu, J. Astr., 02, 448-451 (2007)

13. Y.L. Cai, C.H. Hu, J. Chi. Ine. Tec., 05, 593-597 (2007)

14. W.W. Qin, Z.Q. Zheng, J. Chi. Ine. Tec., 06, 721-724+729 (2008)

15. Y.H. Gao, Y.B. Li, J. Sichuan Uni.(Eng. Sci. Edi.), 02, 177-181 (2010)

16. Y.M. Cai, C.H. Hu. Sys. Eng. Elec., 30(1), 190-192 (2008)

17. Y. Shi, X.M. Li, Com. Meas. \& Con., 21(1), 14-17+26 (2013)

18. Y. Li, Q.F. Luo, J. Shenyang Ligong Uni., 01, 46-51 (2015)

19. S.R. Wang, H. Yang, J. Chi. Ine. Tec., 02, 49-54 (1998)

20. L.N. Xu, Z.L. Deng, J. Chi. Ine. Tec., 01, 50-53+62 (2003)

21. C.L. Fan, Z.H. Jin, J. Shanghai Jiaotong Uni., 01,141-144 (2003)

22. L.Y. Liu, Y.Z. Chen, J. Chi. Ine. Tec., 01, 62-66 (2004)

23. C. Li, J.H. Zhu, J. Pro. Roc. Miss. Gui., SA, $402-404$ (2005)

24. W. Chen, C.H. Hu, Elec. Opt. \& Con., 02, 97-100 (2006)

25. L. Tong, W.M. Niu, Micro. Inf., 10, 159-160+175 (2009)

26. H.M. Qian, W.S. Wang, Nav. Chi., 01, 6-9 (2009)

27. J.T. Wu, H.K. Le, J. Chi. Ine. Tec., 01, 38-40 (1999)

28. Y.J. Lv, C.H. Hu, Mech. Sci. Tec., 01, 28-30 (2006)

29. L. Tong, W.M. Niu, Com. Meas. \& Con., 11, 2137-2139 (2009)

30. L. Yang, W. Zhao, Nav. Con., 09(2), 36-41 (2010)

31. Y.S. Shang, A.Q. Xu, Mech. Sci. Tec., 10, 1711-1715+1720 (2012)

32. M.H. Wu, W.H. Li, Sci. Tec. Eng., 04, 298-302 (2014)

33. J.N. Xu, Y.L. Gu, J. Naval Uni. Eng., 04, 67-70+78 (2001)

34. Z.J. Zhou, C.H. Hu, Sys. Eng. Elec., 03, 416-418 (2007)

35. J. Wang, X.F. Li, Appl. Res. Com., 03, 817-819 (2013)

36. L. Yao, X.P. Han, J. Tele. Trac. Com., 01, 19-23 (2013)

37. Y. Zhang, P.H. Wang, Aero. Shanghai, 03, 22-25 (2005) 